\title{
Decreased immunoreactivity of von Willebrand factor may reflect persistent nature of the endothelial dysfunction in non-ischemic heart failure
}

\author{
Edyta Reichman-Warmusz ${ }^{1}$, Marlena Brzozowa-Zasada ${ }^{1}$, Celina Wojciechowska ${ }^{2}$, \\ Damian Dudek $^{1,3}{ }^{\oplus}$, Oliwia Warmusz ${ }^{1}$, Romuald Wojnicz ${ }^{1}{ }_{(\mathbb{C}}$ \\ ${ }^{1}$ Department of Histology and Cell Pathology, Medical University of Silesia in Katowice, \\ School of Medicine with the Division of Dentistry, Zabrze, Poland \\ ${ }^{2}$ Second Department of Cardiology, Medical University of Silesia, School of Medicine \\ with the Division of Dentistry, Zabrze, Poland \\ ${ }^{3}$ Ambulatory Oral Surgery and Implantology, Torun, Poland
}

\begin{abstract}
Introduction. Endothelial dysfunction is a critical part of heart failure (HF) pathophysiology. It is not clear, however, whether it is present at the similar level in the early and late HF stages.

Material and methods. von Willebrand factor (vWF) and its mRNA levels in biopsies of non-ischemic patients with HF secondary to dilated cardiomyopathy were studied. Consecutive patients with HF were divided into two groups: group A with disease duration $\leq 12$ months $(\mathrm{n}=59)$ and group $\mathrm{B}$ with disease duration $>12$ months $(n=68)$. The immunoreactivity of the vWF was compared with autopsy sections of 19 control cases. Tissue vWF gene expression was analyzed at the mRNA level by RT-PCR.

Results. In the group A, there was lower vWF immunoreactivity in the coronary microvessels compared to the group B [1.5 (1.0-2.0) vs. 2.0 (1.5-2.4), $\mathrm{P}=0.001]$. In the control group, only weak vWF expression was observed. Protein expression was not accompanied by vWF mRNA whose levels were significantly higher in the Group A as compared to the Group B [14671 (4932-51561) vs. 3643 (185.3-9030.8), P = 0.005]. Protein vWF expression was inversely associated with its mRNA levels $(\mathrm{r}=-0.34, \mathrm{P}=0.04)$.

Conclusions. High myocardial protein expression of vWF in patients with long-lasting HF symptoms may highlight the persistent nature of endothelial dysfunction in such a cohort of patients. (Folia Histochemica et Cytobiologica 2021, Vol. 59, No. 2, 108-113)
\end{abstract}

Key words: heart failure; dilated cardiomyopathy; endomyocardial biopsy; endothelial dysfunction; von Willebrand factor; qPCR; IHC

\section{Introduction}

Heart failure is defined as a complex clinical syndrome of impaired heart functions and is a leading cause of morbidity and mortality in developed coun-

Correspondence address: Edyta Reichman-Warmusz, $\mathrm{PhD}$ Department of Histology and Cell Pathology in Zabrze,

Medical University of Silesia in Katowice,

School of Medicine with the Division of Dentistry,

Jordana 19, 41-808 Zabrze, Poland

e-mail: ewarmusz@sum.edu.pl tries. The prevalence of heart failure (HF) worldwide continues to increase, maintaining high rates of morbidity and mortality despite the use of multiple evidence-based therapeutic strategies [1]. The main characteristic of HF is its multifaceted clinical presentation and its progressive nature [2].

For a long time disturbances in the coronary microcirculation have been postulated to contribute to $\mathrm{HF}$ development and progression [3, 4]. It is believed to be a consequence of endothelial dysfunction through loss of its multifaceted regulatory properties [5, 6]. As

This article is available in open access under Creative Common Attribution-Non-Commercial-No Derivatives 4.0 International (CC BY-NC-ND 4.0) license, allowing to download articles and share them with others as long as they credit the authors and the publisher, but without permission to change them in any way or use them commercially. (C) Polish Society for Histochemistry and Cytochemistry 
a result, an abnormal coronary microcirculatory flow may cause impairment of myocardial perfusion and metabolic changes compatible with local myocardial ischemia [7, 8]. More recently it has been reported that endothelial dysfunction in HF independently predicts morbidity and mortality in this cohort of patients $[9,10]$. The exact mechanisms for endothelial dysfunction in HF are unclear. However, it is believed to be the result of lost nitric oxide-dependent vasodilatation, a proinflammatory state, and its prothrombotic properties [11, 12].

Von Willebrand factor (vWF) is a large multimeric glycoprotein produced by endothelial and megakaryocytes that is present in endothelial cells (Weibel-Palade bodies), the subendothelial matrix, platelets and blood plasma [13]. Its main function is mediation of platelet aggregation at the site of vascular injury and thrombus growth. Thus, given the ubiquitous involvement of vWF in local vascular homeostasis, it is not surprising that this factor plays an important role in HF pathogenesis [14]. As vWF release is increased when endothelial cells are injured, it has been proposed as a marker of endothelial dysfunction [15-17]. It is not clear, however, whether endothelial dysfunction is present to the same extent in the HF of short or long duration.

Accordingly, the aim of this study was to clarify this issue by examining biopsy specimens of patients with short and long-standing HF duration by immunohistochemistry (IHC) and RT-qPCR.

\section{Materials and methods}

Patients and material collection. Patients. One hundred and twenty-seven patients with stable, non-ischemic HF (NYHA I to III, LVEF < 40\%), attributable to dilated cardiomyopathy were prospectively studied. Right ventricular endomyocardial biopsy was carried out in all patients. Patients were divided into two groups: Group A with HF duration $\leq 12$ months $(\mathrm{n}=59 ; 50$ men and 9 women $)$, and Group B with HF duration $>12$ months $(\mathrm{n}=68$; 63 men and 5 women). In addition, we used the cardiac autopsy sections of 19 young cases that suddenly died in car crashes (11 men, 8 women, mean age of $33.5 \pm 6.8$ years). This group served as a control for immunohistochemical staining of vWF protein.

All the HF patients had selective coronary angiography to exclude ischemic etiology of HF. In addition, none of the studied patients had other potential causes of HF, including valvular (except relative mitral and/or tricuspid regurgitation), endocrine disease (except impaired glucose tolerance), advanced renal disease, and reported drug or alcohol abuse. All of them were on typical therapeutic regimens for HF including loop diuretics (torasemide and/or furosemide), ACE inhibitors or ATII blockers, $\beta$-blockers, and spironol- actone for at least 6 months prior to the biopsy. Apart from standard HF therapy, none of the patients was treated with oral anticoagulants, statins and antiarrhythmic drugs or had an electrical device implanted before the biopsy was done.

Four to five endomyocardial biopsies were obtained from each patient. All biopsies except one specimen dedicated for molecular biology, were fixed for $20 \mathrm{~min}$ in cold acetone, immersed in embedding medium (OCT Compound, Miles Inc., Sakura Finetek, USA) and cryo-preserved in liquid nitrogen until tested.

This study was carried out in accordance with the Helsinki Declaration, and the study design was approved by the Institutional Ethics Committee (NN-6501-7/07). All patients gave their informed consent.

Immunohistochemistry. For IHC, specimens were cut serially into $5 \mathrm{~mm}$ thick slices on a Cryotome $\mathrm{FSE}^{\circledR}$ Thermo Shandon (Thermo Scientific-Shandon, Lipshaw, PA, USA), air-dried at room temperature and incubated with murine monoclonal anti-vWF antibodies (clone F8/86); dilution 1:300; DAKO). In addition, to exclude myocarditis, frozen sections were incubated with murine monoclonal antihuman antibodies (Abs): ant-HLA-class II (DR antigens), Alpha chain (clone TAL.1B5), anti-HLA-class I (ABC antigens) (clone W6/32), anti-CD3 for T lymphocytes (Clone T34B5), and anti-CD68 (clone EBM11). All antibodies were from DAKO A/S, Glostrup, Denmark. The dilution of the primary antibody was verified in our laboratory in a series of pilot experiments.

The En-Vision method (DAKO En-Vision $\mathrm{Kit}^{\circledR} /$ Alkaline Phosphatase detection system) was used according to the manufacturer's instructions. The bound primary antibody was detected using New Fuchsin Substrate System (DAKO A/S). The primary antibody was omitted from negative control slides. To suppress nonspecific staining due to endogenous alkaline phosphatase activity, Levamisole was used at a final concentration of $0.2 \mathrm{mM}$. The sections were counterstained with Mayer's hematoxylin.

For the semiquantitative assessment of the intensity of vWF microvascular staining, the score index at $200 \times$ magnification was as follows: $(1+)$ weak staining in a few vessels; $(2+)$ moderate staining in most microvessels and $(3+)$ strong staining in all microvessels. All IHC analyses were performed by two investigators independently, blinded to clinical features. The inter-observer variability of immunohistological examinations for vWF was $<3 \%$. Histological results were obtained with the use of Nikon Eclipse $80 i$ microscope with DS-Fi1 digital camera and NIS Elements software from Nikon (Tokyo, Japan).

Quantitative real-time PCR (qPCR). For the real-time PCR examination of the vWF RNA, total RNA was extracted from myocardial specimens by the Trizol ${ }^{\circledR}$ reagent (Invitrogen, Thermo Fisher Scientific, Waltham, MA, USA) 
Table 1. Baseline characteristics of the patients

\begin{tabular}{|c|c|c|c|c|}
\hline & $\begin{array}{c}\text { All patients } \\
\mathbf{n}=127\end{array}$ & $\begin{array}{c}\text { Group A } \\
\mathbf{n}=\mathbf{5 9}\end{array}$ & $\begin{array}{c}\text { Group B } \\
n=68\end{array}$ & $\mathbf{P}$ \\
\hline Age [y] & $44.2 \pm 11.7$ & $40.7 \pm 10.7$ & $42.4 \pm 0.9$ & NS \\
\hline Sex, male/female & $113 / 14$ & $50 / 9$ & $63 / 5$ & NS \\
\hline Time of HF duration [mth] & $29.5 \pm 38.9$ & $7.6 \pm 2.7$ & $52.1 \pm 21.9$ & - \\
\hline Atrial fibrillation, $\mathrm{n}[\%]$ & $21(16.5)$ & $9(15.3)$ & $12(17.6)$ & NS \\
\hline BMI $\left[\mathrm{kg} / \mathrm{m}^{2}\right]$ & $26.3 \pm 4.6$ & $25.5 \pm 4.4$ & $26.2 \pm 5.2$ & NS \\
\hline NYHA class, n, I/II/III & $4 / 110 / 13$ & $3 / 51 / 5$ & $1 / 59 / 8$ & NS \\
\hline LVEF [\%] & $28.1 \pm 7.0$ & $29.2 \pm 6.4$ & $27.6 \pm 7.5$ & NS \\
\hline LVEDD [mm] & $68.6 \pm 10.3$ & $67.5 \pm 11.3$ & $69.6 \pm 0.1$ & NS \\
\hline Serum vWF [\%] & $156.3 \pm 63.5$ & $162.8 \pm 67.3$ & $149.4 \pm 60.3$ & NS \\
\hline Fibrinogen [mg/dL] & $367.2 \pm 95.3$ & $376.0 \pm 107.1$ & $357.9 \pm 81.5$ & NS \\
\hline $\mathrm{CRP}[\mathrm{mg} / \mathrm{dL}]$ & $(-)$ & $1.42(0.14-30.2)$ & $1.64(0.17-21.5)$ & NS \\
\hline $\mathrm{UA}[\mu \mathrm{mol} / \mathrm{L}]$ & $436.9 \pm 111.9$ & $440.5 \pm 114.4$ & $439.0 \pm 114.6$ & NS \\
\hline NT-proBNP $[\mathrm{pg} / \mathrm{mL}]$, median $\left[1^{\left.\text {st }-3^{\text {rd }} \text { quartile }\right]}\right.$ & $898.9(356.5-2338)$ & $886.8(365-3004)$ & $838.3(248.1-2757)$ & NS \\
\hline
\end{tabular}

Values are expressed as mean $\pm \mathrm{SD}$ or as indicated, NS - non-significant. Group A $-\mathrm{HF}$ duration $\leq 12$ mo, group B $-\mathrm{HF}$ duration $>12$ mo. Abbreviations: $\mathrm{BMI}$ - body mass index; CRP — C-reactive protein; HF — heart failure; LVEF - left ventricle ejection fraction; LVEDD — left ventricle end diastolic dimension; NYHA — New York Heart Association; NT-proBNP — amino-terminal pro-B-type natriuretic peptide; UA — uric acid

according to the manufacturer's protocol. The quality of the RNA was assessed by gel electrophoresis and the quantitative analysis of RNA extracts was performed spectrophotometrically using a GeneQuant ${ }^{\mathrm{TM}}$ pro RNA/ /DNA Calculator (Amersham Biosciences, Little Chalfont, United Kingdom). The quantitative analysis was carried out with the use of an Opticon ${ }^{\mathrm{TM}}$ DNA Engine Continuous Fluorescence detector (MJ Research, Reno, $\mathrm{NV}$, United States). The expression of the mRNA for vWF was determined by quantitative real-time PCR (forward sequence: CCTTGAATCCCAGTGACCCTGA, reverse sequence: GGTTCCGAGATGTCCTCCACAT). The PCRs of RNA extracted from biopsy specimens were carried out in 25 cycles. All PCR reactions were terminated by a final elongation step of $10 \mathrm{~min}$ at $72^{\circ} \mathrm{C}$. All samples were tested in triplicate. The PCR amplicons was determined after each round of amplification, using the fluorescent dye SYBR-Green (Sybr Green Quantitect RT-PCR Kit; Qiagen, Venlo, Netherlands). Glyceraldehyde-3-phosphate dehydrogenase (GAPDH) and $\beta$-actin (TaqMan ${ }^{\circledR}$ DNA template reagents kit; PE Applied Biosystems) were included to monitor RT-PCR. To assess vWF gene expression in the studied groups we used the standard curve method to determine the absolute mRNA quantity in the samples. PCR products were separated on $6 \%$ polyacrylamide gel and visualized using silver staining. The length of the amplified fragments was assessed by analysis with Quantity One 4.6.9 software (Molecular Imager ChemiDOC XRS+, BIO-RAD, Hercules, CA, USA).
Statistical analysis. The statistical analysis was made with the SPSS version 16.0 software package (SPSS, Inc., Chicago, IL, USA). Data were analyzed by the Shapiro-Wilk test to determine distribution. Normally distributed data were analyzed using $t$-test (based on normal distribution tested by the Kolmogorov-Smirnov test) and expressed as mean \pm SD. Not normally distributed data are presented as median with interquartile range (IQR; $25^{\text {th }}$ and $75^{\text {th }}$ percentiles). To compare not normally distributed data of vWF immunoreactivity in all studied groups, Kruskal-Wallis analysis of ranks and post hoc test of the Mann-Whitney U test were used. Associations between them were done by the Kendall's tau test. Differences were considered statistically significant at $P<0.05$.

\section{Results}

Clinical and demographic details of the study patients are shown in Table 1. Except for the HF duration, there was no difference in age, gender, etiology of HF, and medications used between the study groups. For the overall group of patients, the average time of symptoms was 19 months (ranging from 0.6 months to 8 years), and all the patients were in NYHA class I, II or III.

In the control cryostat sections, only weak and focally distributed expression of vWF was observed (Fig. 1A and 1B). The cryostat sections taken from HF patients presented both basal and strong immunoreactivity of vWF (Fig. 1C and 1D). In group A (HF duration $\leq 12$ months), there was lower pheno- 

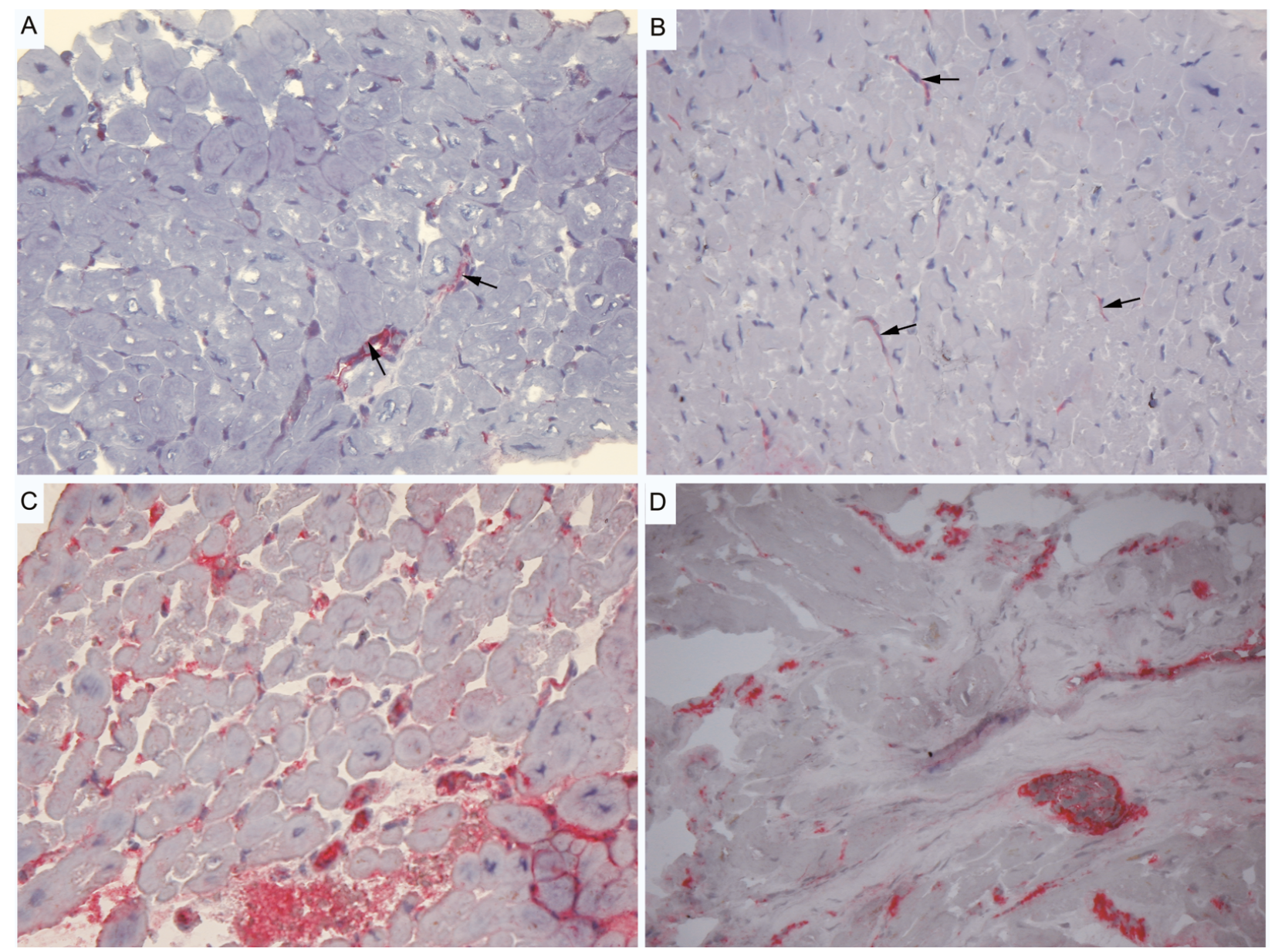

Figure 1. Representative examples of vWF immunostaining in the study groups: Group A - heart failure duration $\leq 12 \mathrm{mths}$, Group B - heart failure duration > $12 \mathrm{mths}$. The basal expression of vWF on sparse microvessels (arrows) in the control subjects (original magnification $100 \times$ ). (C and D) The strong up-regulation of vWF on microvascular endothelium in two HF subjects from the Group B (red color; original magnification $\times 100$ ).

type $\mathrm{vWF}$ expression in the coronary microvessels in comparison with group $\mathrm{B}$ (HF duration $>12$ months) [1.5 (1.0-2.0) vs. 2.0 (1.5-2.4), $\mathrm{P}<0.001]$ (Fig. 2). Unexpectedly, the IHC data was not collaborated with the vWF mRNA copy quantity which were present in a wide range in both study groups and were significantly lower in patients with long-standing disease [median 14671 (range 4932-51561) vs. 3643 (185.3-9030.8), $\mathrm{P}=0.005]$ (Fig. 3). In addition, vWF protein immunoreactivity was inversely associated with $\mathrm{vWF} \mathrm{mRNA}$ levels $(\mathrm{r}=-0.34, \mathrm{P}=0.04)$.

\section{Discussion}

To the best of our knowledge, we have for the first time observed a relationship between up-regulation of $\mathrm{vWF}$ protein immunoreactivity in the myocardium of patients with non-ischemic HF secondary to dilated cardiomyopathy and the disease duration.

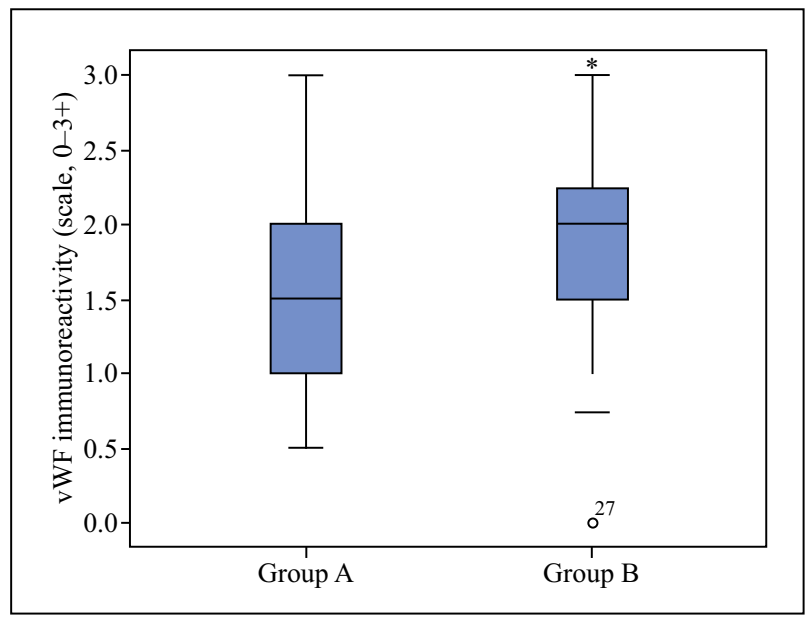

Figure 2. Comparison of the immunoreactivity index (0-3+) for vWF between the Group A (HF duration $\leq 12 \mathrm{mths}$ ) and Group B (HF duration $>12 \mathrm{mths}$ ). Values are medians with IQRs. * $\mathrm{P}<0.001$. The Immunoreactivity index was calculated as described in Methods. 


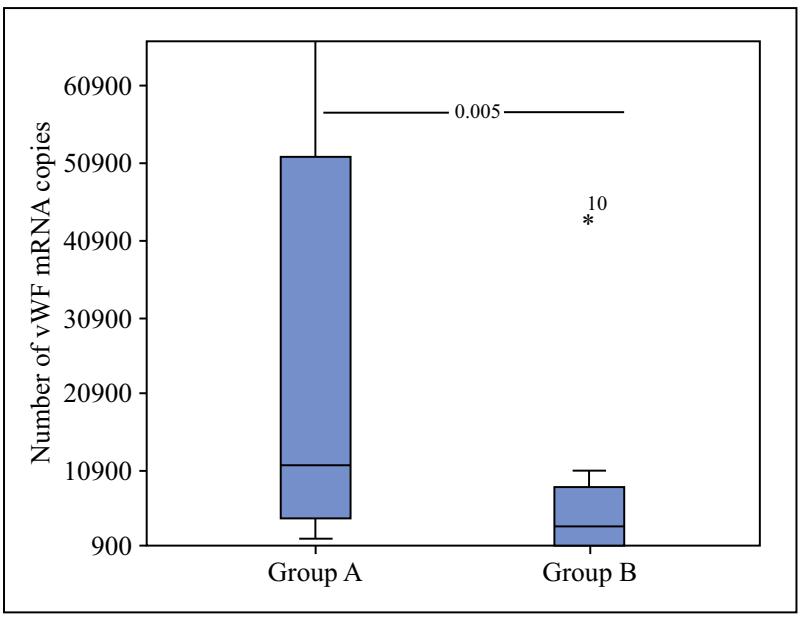

Figure 3. vWF gene expression in the Group A (HF duration $\leq 12$ months) versus Group B (HF duration $>12$ months) by RT-qPCR. Data presented show the mRNA quantity/ $/ \mathrm{g}$ for vWF obtained by the use of standard curve method.

It was known that $\mathrm{vWF}$, stored mainly in the Weibel-Palade bodies of endothelial cells, can be mobilized rapidly after endothelial cell activation [6]. In addition, among hemostatic mediators, $\mathrm{vWF}$ plays a key role in platelet aggregation and stabilization of circulating clotting factors [13].

Most previously published reports focused on soluble vWF levels in plasma of HF patients $[18,19]$. Following the observations by Lip et al., HF were found to substantially increase plasma vWF concentrations and appeared to be highest among patients with acute or recently decompensated HF [20]. Of note, in this study the risk of stroke and thromboembolism was substantially increased in patients with congestive HF concomitant with atrial fibrillation. In another study by Gibbs et al., patients with persistently increased serum concentrations of vWF and higher degree of endothelial dysfunction had a higher risk of thrombosis [21]. Studies by Kleber et al. have shown that plasma vWF was an independent predictor of the long-term outcome in these patients [18].

Little attention has been paid to tissue $\mathrm{vWF}$ expression in HF patients. Fukuchi et al. [22] have provided evidence that stronger tissue immunoreactivity for vWF in the endocardial endothelium in overloaded human atrial appendage may be a local predisposing factor for intra-atrial thrombogenesis. Our findings extend these results, revealing persistent vWF expression on the coronary microvessels in HF patients. Although hypothetical, this may reflect the role of endothelial dysfunction in perpetuating myocardial failure in such a cohort of patients.

The increased $\mathrm{vWF}$ protein immunoreactivity together with diminished levels of mRNA in long-last- ing HF in our study seems to be only on the surface discrepant. Taking into consideration the results of previous study, it is likely that it may reflect lower turnover of this protein rather than an increase in its synthesis in the endothelium. Indeed, it was reported that activity of plasma metalloprotease ADAMTS13 which cleaves vWF was decreased in HF. Such low ADAMTS13 activities were accompanied with high vWF serum levels of HF patients [14].

It has to be kept in mind that the size of sample in our study is limited. Therefore this study may not have been powered enough to verify negative relationship of phenotype protein expression and mRNA levels found. Moreover, we did not perform follow-up biopsies to better reflect time-related changes in protein and mRNA expressions. Our findings cannot be extrapolated to patients with more severe disease or unstable conditions.

Despite these limitations in the present study we demonstrated for the first time the persistent nature of vWF protein expression in the coronary microvessels of non-ischemic HF patients. Considering the results, it may be hypothesized that some of these patients are stranded on a self-perpetuating course of advancing heart failure. Thus, persistent endothelial dysfunction as reflected by the up-regulation of myocardial vWF protein expression in patients with long-standing HF might contribute to local hypercoagulability and hence actively contributes to the disease progression.

\section{Acknowledgment}

This study was supported by grant from Medical University of Silesia, Katowice, Poland (KNW-1066/N/7/O and KNW-1-113/K/8/O).

\section{Conflict of interest}

The authors declare no conflict of interest.

\section{References}

1. Rosamond W, Flegal K, Furie K, et al. American Heart Association Statistics Committee and Stroke Statistics Subcommittee, American Heart Association Statistics Committee and Stroke Statistics Subcommittee. Heart disease and stroke statistics-2008 update: a report from the American Heart Association Statistics Committee and Stroke Statistics Subcommittee. Circulation. 2008; 117(4): e25-146, doi: 10.1161/CIRCULATIONAHA.107.187998, indexed in Pubmed: 18086926.

2. Mosterd A, Hoes AW, Mosterd A, et al. Clinical epidemiology of heart failure. Heart 2007;93:1137-1146. , doi: 10.1136/ hrt.2003.025270, indexed in Pubmed: 17699180.

3. Inoue T, Sakai Y, Morooka S, et al. Coronary flow reserve in patients with dilated cardiomyopathy. Am Heart J. 1993; 125(1): 93-98, doi: 10.1016/0002-8703(93)90061-d, indexed in Pubmed: 8417548.

4. Salgado DR, Favory R, Rocco JR, et al. Microcirculatory effects of angiotensin II inhibitors in patients with severe heart failure. Clin Hemorheol Microcirc. 2013; 54(1): 87-98, doi: 10.3233/CH-2012-1569, indexed in Pubmed: 22890051. 
5. van Hinsbergh VWM. Endothelium-role in regulation of coagulation and inflammation. Semin Immunopathol. 2012; 34(1): 93-106, doi: 10.1007/s00281-011-0285-5, indexed in Pubmed: 21845431.

6. Zuchi C, Tritto I, Carluccio E, et al. Role of endothelial dysfunction in heart failure. Heart Fail Rev. 2020; 25(1): 21-30, doi: 10.1007/s10741-019-09881-3, indexed in Pubmed:31686283.

7. Chen JW, Ting CT, Chen YH, et al. Differential coronary microvascular function in patients with left ventricular dysfunction of unknown cause - implication for possible mechanism of myocardial ischemia in early stage of cardiomyopathy. Int J Cardiol. 1999; 69(3): 251-261, doi: 10.1016/s01675273(99)00042-x, indexed in Pubmed: 10402108.

8. Heuvel Av, Veldhuisen Dv, Wall Ev, et al. Regional myocardial blood flow reserve impairment and metabolic changes suggesting myocardial ischemia in patients with idiopathic dilated cardiomyopathy. J Am Coll Cardiol. 2000; 35(1): 19-28, doi: 10.1016/s0735-1097(99)00499-4, indexed in $\mathrm{Pu}-$ bmed: 10636254 .

9. Neglia D, Michelassi C, Trivieri MG, et al. Prognostic role of myocardial blood flow impairment in idiopathic left ventricular dysfunction. Circulation. 2002; 105(2): 186-193, doi: 10.1161/hc0202.102119, indexed in Pubmed: 11790699.

10. Fischer D, Rossa S, Landmesser U, et al. Endothelial dysfunction in patients with chronic heart failure is independently associated with increased incidence of hospitalization, cardiac transplantation, or death. Eur Heart J. 2005; 26(1): 65-69, doi: 10.1093/eurheartj/ehi001, indexed in Pubmed: 15615801.

11. Katz SD, Hryniewicz K, Hriljac I, et al. Vascular endothelial dysfunction and mortality risk in patients with chronic heart failure. Circulation. 2005; 111(3): 310-314, doi: 10.1161/01. CIR.0000153349.77489.CF, indexed in Pubmed: 15655134.

12. Lerman A, Zeiher A. Endothelial Function. Circulation. 2005; 111(3): 363-368, doi: 10.1161/01.cir.0000153339.27064.14, indexed in Pubmed: 15668353.

13. Ruggeri Z. Structure and Function of von Willebrand Factor. Thromb Haemost. 2017; 82(08): 576-584, doi: 10.1055/s-00371615883, indexed in Pubmed: 10605754.

14. Gombos T, Makó V, Cervenak L, et al. Levels of von Willebrand factor antigen and von Willebrand factor cleaving protease (ADAMTS13) activity predict clinical events in chronic heart failure. Thromb Haemost. 2009; 102(3): 573-580, doi: 10.1160/TH09-01-0036, indexed in Pubmed: 19718479.
15. Nowroozpoor A, Gutterman D, Safdar B. Is microvascular dysfunction a systemic disorder with common biomarkers found in the heart, brain, and kidneys? A scoping review. Microvasc Res. 2021; 134: 104123, doi: 10.1016/j. mvr.2020.104123, indexed in Pubmed: 33333140.

16. Galatius S, Wroblewski H, S rensen V, et al. Endothelin and von Willebrand factor as parameters of endothelial function in idiopathic dilated cardiomyopathy: Different stimuli for release before and after heart transplantation? Am Heart J. 1999; 137(3): 549-554, doi: 10.1016/s0002-8703(99)705053, indexed in Pubmed: 10047640.

17. El-Mansi S, Nightingale TD. Emerging mechanisms to modulate VWF release from endothelial cells. Int J Biochem Cell Biol. 2021; 131: 105900, doi: 10.1016/j.biocel.2020.105900, indexed in Pubmed: 33301925.

18. Kleber ME, Koller L, Goliasch G, et al. Von Willebrand factor improves risk prediction in addition to $\mathrm{N}$-terminal proB-type natriuretic peptide in patients referred to coronary angiography and signs and symptoms of heart failure and preserved ejection fraction. Circ Heart Fail. 2015; 8(1): 25-32, doi: 10.1161/CIRCHEARTFAILURE.114.001478, indexed in Pubmed: 25342739.

19. Zannad F, Stough WG, Regnault V, et al. Is thrombosis a contributor to heart failure pathophysiology? Possible mechanisms, therapeutic opportunities, and clinical investigation challenges. Int J Cardiol. 2013; 167(5): 1772-1782, doi: 10.1016/j.ijcard.2012.12.018, indexed in Pubmed: 23298559.

20. Lip GYH, Pearce LA, Chin BSP, et al. Effects of congestive heart failure on plasma von Willebrand factor and soluble $\mathrm{P}$-selectin concentrations in patients with non-valvar atrial fibrillation. Heart. 2005; 91(6): 759-763, doi: 10.1136/ hrt.2004.036160, indexed in Pubmed: 15894770.

21. Gibbs CR, Blann AD, Watson RD, et al. Abnormalities of hemorheological, endothelial, and platelet function in patients with chronic heart failure in sinus rhythm: effects of angiotensin-converting enzyme inhibitor and beta-blocker therapy. Circulation. 2001; 103(13): 1746-1751, doi: 10.1161/01. cir.103.13.1746, indexed in Pubmed: 11282905.

22. Fukuchi M, Watanabe J, Kumagai K, et al. Increased von Willebrand factor in the endocardium as a local predisposing factor for thrombogenesis in overloaded human atrial appendage. J Am Coll Cardiol. 2001; 37(5): 1436-1442, doi: 10.1016/ s0735-1097(01)01125-1, indexed in Pubmed: 11300458.

Submitted: 30 November, 2020

Accepted after reviews: 29 April, 2021 Available as AoP: 18 May, 2021 